\title{
The $r$-central factorial numbers with even indices
}

\section{F.A. Shiha ${ }^{1 *}$}

\section{"Correspondence:}

fshiha@yahoo.com;

fshiha@mans.edu.eg

${ }^{1}$ Department of Mathematics,

Faculty of Science, Mansoura

University, Mansoura, Egypt

\section{Springer}

\begin{abstract}
In this paper, we introduce the $r$-central factorial numbers with even indices of the first and second kind as extended versions of the central factorial numbers with even indices of both kinds. We obtain several fundamental properties and identities related to these numbers. The connections between the new numbers and the Stirling numbers are presented. In addition, we give the probability distribution of the unsigned $r$-central factorial numbers with even indices. Finally, we consider the $r$-central factorial matrices and study some of their properties.
\end{abstract}

MSC: 05A15; 11B75; 11B83;60C05

Keywords: $r$-central factorial numbers with even indices; Pascal matrix; Stirling numbers

\section{Introduction}

The Stirling numbers of the first kind $s(n, k)$ and of the second kind $S(n, k)$, which are the coefficients of the expansions of factorials into powers and of powers into factorials, respectively, were introduced by J. Stirling [29]:

$$
\begin{aligned}
& (x)_{n}=\sum_{k=0}^{n} s(n, k) x^{k}, \quad n=0,1, \ldots, \\
& x^{n}=\sum_{k=0}^{n} S(n, k)(x)_{k}, \quad n=0,1, \ldots,
\end{aligned}
$$

where $(x)_{n}$ is the falling factorial, i.e., $(x)_{n}=\prod_{i=0}^{n-1}(x-i)$ and $(x)_{0}=1$. The central factorial $x^{[n]}$ is defined by

$$
x^{[n]}=x\left(x+\frac{n}{2}-1\right)_{n-1}, \quad n \geq 1 \text {, with } x^{[0]}=1 .
$$

Riordan [26, pp. 213-217] introduced the central factorial numbers of the first kind $t(n, k)$ and of the second kind $T(n, k)$ as transition coefficients between monomials $x^{n}$ and central

(c) The Author(s) 2020. This article is licensed under a Creative Commons Attribution 4.0 International License, which permits use, sharing, adaptation, distribution and reproduction in any medium or format, as long as you give appropriate credit to the original author(s) and the source, provide a link to the Creative Commons licence, and indicate if changes were made. The images or other third party material in this article are included in the article's Creative Commons licence, unless indicated otherwise in a credit line to the material. If material is not included in the article's Creative Commons licence and your intended use is not permitted by statutory regulation or exceeds the permitted use, you will need to obtain permission directly from the copyright holder. To view a copy of this licence, visit http://creativecommons.org/licenses/by/4.0/. 
factorials $x^{[n]}$ :

$$
\begin{aligned}
& x^{[n]}=\sum_{k=0}^{n} t(n, k) x^{k}, \\
& x^{n}=\sum_{k=0}^{n} T(n, k) x^{[k]},
\end{aligned}
$$

with $t(n, 0)=T(n, 0)=\delta_{n, 0}$, where $\delta_{n, k}$ is the Kronecker delta: $\delta_{n, n}=1, \delta_{n, k}=0$ for $n \neq k$. Note that if $n$ and $k$ have opposite parity (one is odd, the other is even), then $t(n, k)=$ $T(n, k)=0$; and if $n$ and $k$ are both odd, then $t(n, k)$ and $T(n, k)$ are not integers.

Kim and Kim [16] considered the central Bell polynomials $B_{n}^{(c)}(x)$ and the central Bell numbers $B_{n}^{(c)}$ associated with the central factorial numbers of the second kind:

$$
\begin{aligned}
& B_{n}^{(c)}(x)=\sum_{k=o}^{n} x^{k} T(n, k) \quad(n \geq 0), \\
& B_{n}^{(c)}=B_{n}^{(c)}(1)=\sum_{k=o}^{n} T(n, k) \quad(n \geq 0) .
\end{aligned}
$$

The central Bell polynomials and the central factorial numbers of the second kind were extended to the central complete and incomplete Bell polynomials. For more details, see [19].

In recent years, many mathematicians introduced and studied various degenerate and extended versions of a lot of old and new special numbers and polynomials, namely Bernoulli numbers and polynomials, Eulerian numbers and polynomials, Daehee numbers, Bell polynomials, and type 2 Bernoulli polynomials of the second kind, to name a few (see $[1,2,11,15,17,28]$ and the references therein). Here, we are interested in extended versions of the central factorial numbers.

For any nonnegative integer $r$, Kim et al. [20] defined the extended central factorial numbers of the second kind $T^{(r)}(n, k)$ :

$$
\frac{1}{k !}\left(e^{\frac{t}{2}}-e^{\frac{-t}{2}}+r t\right)^{k}=\sum_{n=k}^{\infty} T^{(r)}(n, k) \frac{t^{n}}{n !}
$$

Kim et al. [12] introduced the extended $r$-central factorial numbers of the second kind $T_{r}(n+r, k+r)$ and the extended $r$-central Bell polynomials $B_{n}^{(c, r)}(x)$ as extended versions of $T(n, k)$ and $B_{n}^{(c)}(x)$, respectively. The numbers $T_{r}(n+r, k+r)$ are either given by

$$
\frac{1}{k !} e^{r t}\left(e^{\frac{t}{2}}-e^{\frac{-t}{2}}\right)^{k}=\sum_{n=k}^{\infty} T_{r}(n+r, k+r) \frac{t^{n}}{n !}
$$

or given by

$$
(x+r)^{n}=\sum_{k=0}^{n} T_{r}(n+r, k+r) x^{[k]}
$$


and

$$
B_{n}^{(c, r)}(x)=\sum_{k=0}^{n} x^{k} T_{r}(n+r, k+r) .
$$

For more details and further properties and identities related to these numbers and polynomials using umbral calculus techniques, see [10].

Degenerate versions, incomplete and complete versions, and degenerate complete and incomplete versions of $T_{r}(n+r, k+r)$ and $B_{n}^{(c, r)}(x)$ were introduced and studied in [13, 14, $18]$, and [21], respectively.

Recall that the central factorial numbers with even indices of the first and second kind, respectively, are denoted by

$$
u(n, k)=t(2 n, 2 k) \quad \text { and } \quad U(n, k)=T(2 n, 2 k) \quad(\text { see }[8])
$$

They satisfy the recurrence relations

$$
\begin{aligned}
& u(n, k)=u(n-1, k-1)-(n-1)^{2} u(n-1, k), \quad n \geq k \geq 1, \\
& U(n, k)=U(n-1, k-1)+k^{2} U(n-1, k), \quad n \geq k \geq 1
\end{aligned}
$$

The explicit formula of $U(n, k)$ is given by

$$
U(n, k)=\frac{2}{(2 k) !} \sum_{j=1}^{k}(-1)^{k+j}\left(\begin{array}{c}
2 k \\
k-j
\end{array}\right) j^{2 n} \quad(\text { see [4] })
$$

The combinatorial interpretations of $u(n, k)$ and $U(n, k)$ can be found in [8]. See [24] for the connections between these numbers and Bernoulli polynomials.

In this paper, we consider the $r$-central factorial numbers with even indices of the first and second kind, which we will denote by $u_{r}(n, k)$ and $U_{r}(n, k)$, respectively. We study various properties and identities related to these numbers. In addition, we give some explicit formulas for these numbers. Finally, we represent $u_{r}(n, k)$ and $U_{r}(n, k)$ in terms of $s(n, k)$ and $S(n, k)$, respectively.

\section{The $r$-central factorial numbers with even indices}

Definition 1 The arrays $u_{r}(n, k)$ and $U_{r}(n, k)$ for nonnegative integers $r, n$, and $k$ with $n \geq k \geq 0$ are determined by the recurrences

$$
\begin{aligned}
& u_{r}(n, k)=u_{r}(n-1, k-1)-\left((n-1)^{2}+r\right) u_{r}(n-1, k), \quad n, k \geq 1, \\
& U_{r}(n, k)=U_{r}(n-1, k-1)+\left(k^{2}+r\right) U_{r}(n-1, k), \quad n, k \geq 1,
\end{aligned}
$$

with initial values $u_{r}(n, 0)=(-1)^{n} \prod_{i=0}^{n-1}\left(i^{2}+r\right), U_{r}(n, 0)=r^{n}$, and $u_{r}(0, k)=U_{r}(0, k)=\delta_{k, 0}$ for all $n, k \geq 0$.

Note that at $r=0$, these numbers are reduced to the central factorial numbers with even indices, i.e., $u_{0}(n, k)=u(n, k)$ and $U_{0}(n, k)=U(n, k)$. From (9) and (10), it is easy to observe 
that

$$
\begin{aligned}
& u_{r}(n, 1)=(-1)^{n-1} \prod_{\ell=0}^{n-1}\left(\ell^{2}+r\right) \sum_{i=0}^{n-1} \frac{1}{r+i^{2}}, \quad U_{r}(n, 1)=(r+1)^{n}-r^{n}, \\
& u_{r}(n, n-1)=-\sum_{\ell=0}^{n-1}\left(r+\ell^{2}\right), \quad U_{r}(n, n-1)=\sum_{\ell=0}^{n-1}\left(r+\ell^{2}\right), \\
& u_{r}(n, n)=1, \quad U_{r}(n, n)=1 .
\end{aligned}
$$

We next show that $u_{r}(n, k)$ and $U_{r}(n, k)$ can be defined as connection coefficients between some special polynomials.

Theorem 1 For $n \geq 0$, then

$$
\begin{aligned}
& \prod_{i=0}^{n-1}\left(x-i^{2}\right)=\sum_{k=0}^{n} u_{r}(n, k)(x+r)^{k}, \\
& (x+r)^{n}=\sum_{k=0}^{n} U_{r}(n, k) \prod_{i=0}^{k-1}\left(x-i^{2}\right) .
\end{aligned}
$$

Proof We prove (11) by induction on $n$ and (12) is proven similarly, the initial case of $n=0,1$ being obvious. Suppose that the statement is true for $n$, we prove it for $n+1$ :

$$
\begin{aligned}
& \sum_{k=0}^{n+1} u_{r}(n+1, k)(x+r)^{k} \\
& \quad=\sum_{k=0}^{n} u_{r}(n+1, k)(x+r)^{k}+(x+r)^{n+1} \\
& =\sum_{k=0}^{n} u_{r}(n, k-1)(x+r)^{k}-\left(n^{2}+r\right) \sum_{k=0}^{n} u_{r}(n, k)(x+r)^{k}+(x+r)^{n+1} \\
& =\sum_{k=0}^{n-1} u_{r}(n, k)(x+r)^{k+1}-\left(n^{2}+r\right) \prod_{i=0}^{n-1}\left(x-i^{2}\right)+(x+r)^{n+1} \\
& =\sum_{k=0}^{n} u_{r}(n, k)(x+r)^{k+1}-(x+r)^{n+1}-\left(n^{2}+r\right) \prod_{i=0}^{n-1}\left(x-i^{2}\right)+(x+r)^{n+1} \\
& =(x+r) \prod_{i=0}^{n-1}\left(x-i^{2}\right)-\left(n^{2}+r\right) \prod_{i=0}^{n-1}\left(x-i^{2}\right) \\
& =\prod_{i=0}^{n-1}\left(x-i^{2}\right)\left(x-n^{2}\right)=\prod_{i=0}^{n}\left(x-i^{2}\right),
\end{aligned}
$$

which completes the induction.

Remark 1 From (11) and (12), we get the following orthogonal relation:

$$
\sum_{k=i}^{n} u_{r}(n, k) U_{r}(k, i)=\sum_{k=i}^{n} U_{r}(n, k) u_{r}(k, i)=\delta_{n, i}
$$


In the following theorem, we derive an explicit formula for the array $U_{r}(n, k)$ from the Newton interpolation formula.

Theorem 2 For any integer $0 \leq k \leq n$,

$$
U_{r}(n, k)=\frac{2}{(2 k) !} \sum_{j=0}^{k}(-1)^{k+j}\left(\begin{array}{c}
2 k \\
k-j
\end{array}\right)\left(j^{2}+r\right)^{n} \text {. }
$$

Proof Using the Newton interpolation formula, we have

$$
x^{n}=\sum_{k=0}^{n}\left(\sum_{j=0}^{k} \frac{x_{j}^{n}}{\prod_{i=0, i \neq j}^{k}\left(x_{j}-x_{i}\right)}\right) \prod_{i=0}^{k-1}\left(x-x_{i}\right) .
$$

Then replacing $x$ by $x+r$ and $x_{i}$ by $i^{2}+r$, we get

$$
(x+r)^{n}=\sum_{k=0}^{n}\left(\sum_{j=0}^{k} \frac{\left(j^{2}+r\right)^{n}}{\prod_{i=0, i \neq j}^{k}\left(j^{2}-i^{2}\right)}\right) \prod_{i=0}^{k-1}\left(x-i^{2}\right) .
$$

Then

$$
\begin{aligned}
U_{r}(n, k) & =\sum_{j=0}^{k} \frac{\prod_{i=0}^{n-1}\left(j^{2}+r\right)}{\prod_{i=0, i \neq j}^{k}\left(j^{2}-i^{2}\right)}=\sum_{j=0}^{k} \frac{2(-1)^{k+j}\left(j^{2}+r\right)^{n}}{(k-j) !(k+j) !} \\
& =\frac{2}{(2 k) !} \sum_{j=0}^{k}(-1)^{k+j}\left(\begin{array}{c}
2 k \\
k-j
\end{array}\right)\left(j^{2}+r\right)^{n} .
\end{aligned}
$$

Multiplying both sides of (14) by $\frac{t^{n}}{n !}$ and summing over $n \geq k$ gives the exponential generating function of $U_{r}(n, k)$ :

$$
\sum_{n=k}^{\infty} U_{r}(n, k) \frac{t^{n}}{n !}=\frac{2}{(2 k) !} \sum_{j=0}^{k}(-1)^{k+j}\left(\begin{array}{c}
2 k \\
k-j
\end{array}\right) e^{\left(j^{2}+r\right) t}
$$

In particular, at $r=0$, we get the exponential generating function of $U(n, k)$ :

$$
\sum_{n=k}^{\infty} U(n, k) \frac{t^{n}}{n !}=\frac{2}{(2 k) !} \sum_{j=0}^{k}(-1)^{k+j}\left(\begin{array}{c}
2 k \\
k-j
\end{array}\right) e^{j^{2} t}
$$

\section{Log-concavity and distribution of $\left|u_{r}(n, k)\right|$}

A sequence $\left\{a_{i}\right\}_{i=0}^{n}$ of real numbers is said to be log-concave (strict log-concave) if $\left(a_{k}\right)^{2} \geq$ $a_{k-1} a_{k+1}\left(\left(a_{k}\right)^{2}>a_{k-1} a_{k+1}\right)$ for any $k \geq 1$; it is said to be unimodal if there exists an index $0 \leq j \leq n$ such that $c_{i} \leq c_{i+1}$ for $i=0, \ldots, j-1$ and $c_{i} \geq c_{i+1}$ for $i=j, \ldots, n-1$. Clearly, a log-concave sequence of positive terms is unimodal (see [3]).

Proposition 1 (Wilf [30]) Let $\sum_{i=0}^{n} a_{i} x^{i}$ be a polynomial with positive coefficients and with only real and negative zeros. Then the sequence $\left\{a_{i}\right\}_{i=0}^{n}$ is strictly log-concave and it is also unimodal. 
The unsigned $r$-central factorial numbers of even indices of the first kind are defined by

$$
\mathbf{u}_{r}(n, k)=(-1)^{n-k} u_{r}(n, k)=\left|u_{r}(n, k)\right|
$$

Theorem 3 For any fixed positive integer $n$, the sequence $\left\{\mathbf{u}_{r}(n, k)\right\}_{k=0}^{n}$ is strictly log-concave (and thus unimodal).

Proof Replacing $x$ by $-x-r$ in (11), we get the polynomial

$$
\sum_{k=0}^{n} \mathbf{u}_{r}(n, k) x^{k}=\prod_{j=0}^{n-1}\left(x+r+j^{2}\right)=(x+r)\left(x+r+1^{2}\right) \cdots\left(x+r+(n-1)^{2}\right),
$$

whose zeros are real and negative. Proposition 1 implies that the sequence $\left\{\mathbf{u}_{r}(n, k)\right\}_{k=0}^{n}$ is strictly log-concave.

As consequences of Theorem 3, the sequence $\left\{\mathbf{u}_{r}(n, k)\right\}_{k=0}^{n}$ satisfies the inequalities

$$
\left(\mathbf{u}_{r}(n, k)\right)^{2}>\mathbf{u}_{r}(n, k-1) \mathbf{u}_{r}(n, k+1), \quad k=1, \ldots, n-1 .
$$

Theorem 4 The array $\mathbf{u}_{r}(n, k)$ is Poisson-binomially distributed.

Proof Let us define random variables $Y_{n}, n=1,2, \ldots$, such that

$$
P\left(Y_{n}=k\right)=\frac{\mathbf{u}_{r}(n, k)}{\sum_{k=0}^{n} \mathbf{u}_{r}(n, k)}=\frac{\mathbf{u}_{r}(n, k)}{\prod_{i=0}^{n-1}\left(1+r+i^{2}\right)}, \quad k=0,1, \ldots n .
$$

The probability generating function of $Y_{n}$ is given by

$$
\begin{aligned}
\mathrm{E}\left(s^{Y_{n}}\right) & =\sum_{k=0}^{n} s^{k} P\left(Y_{n}=k\right)=\prod_{i=0}^{n-1} \frac{s+r+i^{2}}{1+r+i^{2}} \\
& =\prod_{i=0}^{n-1}\left(1-\frac{1}{1+r+i^{2}}+\frac{s}{1+r+i^{2}}\right) .
\end{aligned}
$$

Then $Y_{n}$ can be represented as a sum of independent zero-one Bernoulli random variables $X_{0}, X_{1}, \ldots, X_{n-1}$ with probabilities $p_{i}$ of success on the $i$ th trial:

$$
p_{i}=P\left(X_{i}=1\right)=1-P\left(X_{i}=0\right)=\frac{1}{1+r+i^{2}},
$$

and then the random variable $Y_{n}=\sum_{i=0}^{n-1} X_{i}$ has a Poisson-binomial distribution (which is a generalization of the binomial distribution) with mean and variance given by

$$
\begin{aligned}
& \mathrm{E}\left(Y_{n}\right)=\sum_{i=0}^{n-1} p_{i}=\sum_{i=0}^{n-1} \frac{1}{1+r+i^{2}}, \\
& \operatorname{Var}\left(Y_{n}\right)=\sum_{i=0}^{n-1} p_{i}\left(1-p_{i}\right)=\sum_{i=0}^{n-1} \frac{r+i^{2}}{\left(1+r+i^{2}\right)^{2}} .
\end{aligned}
$$


Note from (20) that $P\left(Y_{n}=k\right)$ differs from the array $\mathbf{u}_{r}(n, k)$ only by a normalizing constant, and thus completely characterizes the distribution of $\mathbf{u}_{r}(n, k)$.

Using the same previous assumption, one can get an alternative proof of Theorem 3 as follows:

Let $f_{n}(k)=P\left(Y_{n}=k\right)$ be the probability distribution function of $Y_{n}$ defined in (20), equation (21) can be rewritten in the form

$$
\sum_{k=0}^{n} f_{n}(k) s^{k}=\prod_{i=0}^{n-1}\left(1-p_{i}+p_{i} s\right)
$$

An inequality of Newton found in [9, p. 104] and [27] states that if $\left\{a_{i}\right\}_{i=0}^{n-1}$ are any nonzero real numbers (positive or negative) and if $\left\{b_{i}\right\}_{i=0}^{n}$ are defined by

$$
\sum_{k=0}^{n}\left(\begin{array}{l}
n \\
k
\end{array}\right) b_{k} s^{k}=\prod_{i=0}^{n-1}\left(1+a_{i} s\right),
$$

then

$$
b_{k}^{2}>b_{k-1} b_{k+1} \quad \text { for } k=1,2, \ldots, n \text {. }
$$

Setting $b_{k}=\frac{f_{n}(k)}{\left(\begin{array}{c}n \\ k\end{array}\right)}$, we obtain

$$
\left(\frac{f_{n}(k)}{\left(\begin{array}{l}
n \\
k
\end{array}\right)}\right)^{2}>\left(\frac{f_{n}(k-1)}{\left(\begin{array}{c}
n \\
k-1
\end{array}\right)}\right)\left(\frac{f_{n}(k+1)}{\left(\begin{array}{c}
n \\
k+1
\end{array}\right)}\right) .
$$

So, we have the inequality

$$
\left(f_{n}(k)\right)^{2}>f_{n}(k-1) f_{n}(k+1)
$$

that is, $f_{n}(k)$ is strictly long-concave. Since $f_{n}(k)=\mathbf{u}_{r}(n, k) \prod_{i=0}^{n-1} p_{i}$, and $\prod_{i=0}^{n-1} p_{i}$ is clearly strict log-concave, then $\mathbf{u}_{r}(n, k)$ also.

\section{Identities of the $r$-central factorial numbers with even indices}

Theorem 5 For fixed $n \geq 0$, the generating functions of the arrays $u_{r}(n, k)$ and $U_{r}(n, k)$ are given, respectively, by

$$
\begin{aligned}
& \sum_{k=0}^{n} u_{r}(n, n-k) x^{k}=\prod_{k=0}^{n-1}\left(1-\left(k^{2}+r\right) x\right), \\
& \sum_{n=k}^{\infty} U_{r}(n, k) t^{n}=\frac{t^{k}}{\prod_{j=0}^{k}\left(1-\left(j^{2}+r\right) t\right)}, \quad k \geq 0 .
\end{aligned}
$$


Proof We prove (27) by induction on $n$. The equality holds for $n=0$. Assume that the equality holds for $n$, and we prove it for $n+1$. From recurrence (9), we have

$$
\begin{aligned}
\sum_{k=0}^{n+1} u_{r}(n+1, n+1-k) x^{k} & \\
\quad= & \sum_{k=0}^{n} u_{r}(n, n-k) x^{k}-\left(n^{2}+r\right) \sum_{k=0}^{n+1} u_{r}(n, n+1-k) x^{k} \\
= & \prod_{k=0}^{n-1}\left(1-\left(k^{2}+r\right) x\right)-\left(n^{2}+r\right) \sum_{k=0}^{n} u_{r}(n, n-k) x^{k+1} \\
= & \prod_{k=0}^{n-1}\left(1-\left(k^{2}+r\right) x\right)-\left(n^{2}+r\right) x \prod_{k=0}^{n-1}\left(1-\left(k^{2}+r\right) x\right) \\
= & \prod_{k=0}^{n-1}\left(1-\left(k^{2}+r\right) x\right)\left(1-\left(n^{2}+r\right) x\right)=\prod_{k=0}^{n}\left(1-\left(k^{2}+r\right) x\right)
\end{aligned}
$$

which completes the induction. For (28), let $U_{r}^{(k)}(t)=\sum_{n \geq k} U_{r}(n, k) t^{n}$, then the initial condition is given by

$$
U_{r}^{(0)}(t)=\sum_{n \geq 0} U_{r}(n, 0) t^{n}=\sum_{n \geq 0}(r t)^{n}=\frac{1}{1-r t}
$$

Multiplying both sides of (10) by $t^{n}$ and summing over $n \geq k$ gives

$$
U_{r}^{(k)}(t)=t U_{r}^{(k-1)}(t)+\left(k^{2}+r\right) t U_{r}^{(k)}(t)
$$

Then

$$
U_{r}^{(k)}(t)=\frac{t}{1-\left(k^{2}+r\right) t} U_{r}^{(k-1)}(t), \quad k \geq 1
$$

Iterating this recurrence gives

$$
U_{r}^{(k)}(t)=U_{r}^{(0)}(t) \frac{t}{1-(1+r) t} \frac{t}{1-\left(2^{2}+r\right) t} \cdots \frac{t}{1-\left(k^{2}+r\right) t},
$$

and according to initial condition (29), we deduce (28).

Given a set of variables $z_{1}, z_{2}, \ldots, z_{n}$, the $k$ th elementary symmetric function $\sigma_{k}\left(z_{1}, z_{2}, \ldots\right.$, $z_{n}$ ) is the sum of all possible products of $k$ of these $n$ variables, chosen without replacement, i.e.,

$$
\sigma_{k}\left(z_{1}, z_{2}, \ldots, z_{n}\right)=\sum_{1 \leq j_{1}<j_{2}<\cdots<j_{k} \leq n} z_{j_{1}} z_{j_{2}} \cdots z_{j_{k}}, \quad 1 \leq k \leq n .
$$

If the choice is with replacement, then we have the $k$ th complete homogeneous symmetric function $h_{k}\left(z_{1}, z_{2}, \ldots, z_{n}\right)$ :

$$
h_{k}\left(z_{1}, z_{2}, \ldots, z_{n}\right)=\sum_{1 \leq j_{1} \leq j_{2} \leq \cdots \leq j_{k} \leq n} z_{j_{1}} z_{j_{2}} \cdots z_{j_{k}}, \quad k \geq 1,
$$


with initial conditions $\sigma_{0}\left(z_{1}, z_{2}, \ldots, z_{n}\right)=h_{0}\left(z_{1}, z_{2}, \ldots, z_{n}\right)=1$. Note that $\sigma_{k}\left(z_{1}, z_{2}, \ldots, z_{n}\right)=0$ for $k>n$ and $h_{k}\left(z_{1}, z_{2}, \ldots, z_{n}\right) \neq 0$ for $k>n$, for example $h_{3}\left(z_{1}, z_{2}\right)=z_{1}^{3}+z_{1}^{2} z_{2}+z_{1} z_{2}^{2}+z_{2}^{3}$. The generating functions for $\sigma_{k}$ and $h_{k}$ are given by

$$
\begin{aligned}
\sum_{k=0}^{n} \sigma_{k}\left(z_{1}, z_{2}, \ldots, z_{n}\right) t^{k} & =\prod_{i=1}^{n}\left(1+z_{i} t\right), \\
\sum_{k \geq 0} h_{k}\left(z_{1}, z_{2}, \ldots, z_{n}\right) t^{k} & =\prod_{i=1}^{n}\left(1-z_{i} t\right)^{-1} .
\end{aligned}
$$

From (27) and (28), we deduce that the numbers $u_{r}(n, k)$ and $U_{r}(n, k)$ are the specializations of the elementary and complete symmetric functions given by

$$
\begin{aligned}
& u_{r}(n, n-k)=(-1)^{k} \sigma_{k}\left(r, 1^{2}+r, 2^{2}+r, \ldots,(n-1)^{2}+r\right), \\
& U_{r}(n+k, n)=h_{k}\left(r, 1^{2}+r, 2^{2}+r, \ldots, n^{2}+r\right) .
\end{aligned}
$$

Recall that the central factorial numbers with even indices of both kinds satisfy

$$
\begin{aligned}
& u(n, n-k)=(-1)^{k} \sigma_{k}\left(1^{2}, 2^{2}, \ldots,(n-1)^{2}\right), \\
& U(n+k, n)=h_{k}\left(1^{2}, 2^{2}, \ldots, n^{2}\right) .
\end{aligned}
$$

And the Stirling numbers of both kinds satisfy

$$
\begin{aligned}
& s(n, n-k)=(-1)^{k} \sigma_{k}(1,2, \ldots, n-1), \\
& S(n+k, n)=h_{k}(1,2, \ldots, n) .
\end{aligned}
$$

Proposition 2 (Merca [23]) Let $k$ and $n$ be two positive integers, then

$$
g_{k}\left(z_{1}+t, z_{2}+t, \ldots, z_{n}+t\right)=\sum_{i=0}^{k}\left(\begin{array}{c}
n-c_{i} \\
k-i
\end{array}\right) g_{i}\left(z_{1}, z_{2}, \ldots, z_{n}\right) t^{k-i},
$$

where $t, z_{1}, z_{2}, \ldots, z_{n}$ are variables, $g_{i}$ is any of these complete or elementary symmetric functions and

$$
c_{i}= \begin{cases}i, & \text { if } g_{i}=\sigma_{i}, \\ 1-k, & \text { if } g_{i}=h_{i} .\end{cases}
$$

In the next theorem, we show that the array $u_{r}(n, k)\left(U_{r}(n, k)\right)$ can be expressed in terms of $u(n, k)(U(n, k))$ and vice versa.

Theorem 6 If $n, k, r \geq 0$, then

$$
\begin{aligned}
& u_{r}(n, k)=\sum_{i=k}^{n}\left(\begin{array}{l}
i \\
k
\end{array}\right) u(n, i)(-r)^{i-k}, \\
& u(n, k)=\sum_{i=k}^{n}\left(\begin{array}{l}
i \\
k
\end{array}\right) u_{r}(n, i) r^{i-k},
\end{aligned}
$$




$$
\begin{aligned}
& U_{r}(n, k)=\sum_{i=k}^{n}\left(\begin{array}{l}
n \\
i
\end{array}\right) U(i, k) r^{n-i}, \\
& U(n, k)=\sum_{i=k}^{n}\left(\begin{array}{l}
n \\
i
\end{array}\right) U_{r}(i, k)(-r)^{n-i} .
\end{aligned}
$$

Proof To show (40), note that

$$
\begin{aligned}
u_{r}(n, n-k) & =(-1)^{k} \sigma_{k}\left(r, 1^{2}+r, 2^{2}+r, \ldots,(n-1)^{2}+r\right) \\
& =(-1)^{k} \sum_{i=0}^{k}\left(\begin{array}{l}
n-i \\
k-i
\end{array}\right) \sigma_{i}\left(0,1^{2}, \ldots,(n-1)^{2}\right) r^{k-i} \\
& =\sum_{i=0}^{k}\left(\begin{array}{l}
n-i \\
k-i
\end{array}\right)(-1)^{i} \sigma_{i}\left(0,1^{2}, \ldots,(n-1)^{2}\right)(-r)^{k-i} \\
& =\sum_{i=0}^{k}\left(\begin{array}{l}
n-i \\
k-i
\end{array}\right) u(n, n-i)(-r)^{k-i} .
\end{aligned}
$$

Then

$$
\begin{aligned}
u_{r}(n, k) & =\sum_{i=0}^{n-k}\left(\begin{array}{c}
n-i \\
n-k-i
\end{array}\right) u(n, n-i)(-r)^{n-k-i} \\
& =\sum_{i=k}^{n}\left(\begin{array}{c}
i \\
i-k
\end{array}\right) u(n, i)(-r)^{i-k} .
\end{aligned}
$$

For (42), note that

$$
\begin{aligned}
U_{r}(n+k, n) & =h_{k}\left(r, 1^{2}+r, \ldots, n^{2}+r\right) \\
& =\sum_{i=0}^{k}\left(\begin{array}{c}
n+k \\
k-i
\end{array}\right) h_{i}\left(0^{2}, 1^{2}, \ldots, n^{2}\right) r^{k-i} \\
& =\sum_{i=0}^{k}\left(\begin{array}{l}
n+k \\
k-i
\end{array}\right) U(n+i, n) r^{k-i} .
\end{aligned}
$$

Thus

$$
U_{r}(n, n-k)=\sum_{i=0}^{k}\left(\begin{array}{c}
n \\
k-i
\end{array}\right) U(n-k+i, n-k) r^{k-i}
$$

then we obtain

$$
U_{r}(n, k)=\sum_{i=0}^{n-k}\left(\begin{array}{c}
n \\
n-k-i
\end{array}\right) U(k+i, k) r^{n-k-i}=\sum_{i=k}^{n}\left(\begin{array}{c}
n \\
n-i
\end{array}\right) U(i, k) r^{n-i}
$$

The proofs of (41) and (43) are similar. 
Merca [22] showed that

$$
\sigma_{k}\left(z_{1}^{2}, z_{2}^{2}, \ldots, z_{n}^{2}\right)=\sum_{i=-k}^{k}(-1)^{i} \sigma_{k-i}\left(z_{1}, z_{2}, \ldots, z_{n}\right) \sigma_{k+i}\left(z_{1}, z_{2}, \ldots, z_{n}\right)
$$

Similarly, we can deduce combinatorial identity for $h_{k}\left(z_{1}^{2}, z_{2}^{2}, \ldots, z_{n}^{2}\right)$.

Theorem 7 Let $k$ and $n$ be positive integers. Then

$$
h_{k}\left(z_{1}^{2}, z_{2}^{2}, \ldots, z_{n}^{2}\right)=\sum_{i=-k}^{k}(-1)^{k-i} h_{k-i}\left(z_{1}, z_{2}, \ldots, z_{n}\right) h_{k+i}\left(z_{1}, z_{2}, \ldots, z_{n}\right)
$$

Proof It is clear that $h_{k}\left(-z_{1},-z_{2}, \ldots,-z_{n}\right)=(-1)^{k} h_{k}\left(z_{1}, z_{2}, \ldots, z_{n}\right)$, hence

$$
\sum_{k=0}^{\infty}(-1)^{k} h_{k}\left(z_{1}, z_{2}, \ldots, z_{n}\right) t^{k}=\prod_{i=1}^{n}\left(1+z_{i} t\right)^{-1}
$$

Replacing $z_{i}$ by $z_{i}^{2}$ and $t$ by $t^{2}$ in (32), we get

$$
\sum_{k=0}^{\infty} h_{k}\left(z_{1}^{2}, z_{2}^{2}, \ldots, z_{n}^{2}\right) t^{2 k}=\prod_{i=1}^{n}\left(1-z_{i}^{2} t^{2}\right)^{-1}
$$

and we can write

$$
\begin{aligned}
\prod_{i=1}^{n}\left(1-z_{i}^{2} t^{2}\right)^{-1} & =\prod_{i=1}^{n}\left(1+z_{i} t\right)^{-1} \prod_{i=1}^{n}\left(1-z_{i} t\right)^{-1} \\
& =\sum_{k=0}^{\infty}(-1)^{k} h_{k}\left(z_{1}, z_{2}, \ldots, z_{n}\right) t^{k} \sum_{k=0}^{\infty} h_{k}\left(z_{1}, z_{2}, \ldots, z_{n}\right) t^{k} \\
& =\sum_{k=0}^{\infty} \sum_{i=0}^{k}(-1)^{i} h_{i}\left(z_{1}, z_{2}, \ldots, z_{n}\right) h_{k-i}\left(z_{1}, z_{2}, \ldots, z_{n}\right) t^{k} \\
& =\sum_{k=0}^{\infty}\left(\sum_{i=0}^{2 k}(-1)^{i} h_{i}\left(z_{1}, z_{2}, \ldots, z_{n}\right) h_{2 k-i}\left(z_{1}, z_{2}, \ldots, z_{n}\right)\right) t^{2 k} .
\end{aligned}
$$

By (46), we obtain

$$
\begin{aligned}
h_{k}\left(z_{1}^{2}, z_{2}^{2}, \ldots, z_{n}^{2}\right) & =\sum_{i=0}^{2 k}(-1)^{i} h_{i}\left(z_{1}, z_{2}, \ldots, z_{n}\right) h_{2 k-i}\left(z_{1}, z_{2}, \ldots, z_{n}\right) \\
& =\sum_{i=-k}^{k}(-1)^{k-i} h_{k-i}\left(z_{1}, z_{2}, \ldots, z_{n}\right) h_{k+i}\left(z_{1}, z_{2}, \ldots, z_{n}\right) .
\end{aligned}
$$

Using the prior identities, we show in the next theorem the connection between the $r$-central factorial numbers with even indices of both kinds and the Stirling numbers. 
Theorem 8 If $n, k, r \geq 0$, then

$$
\begin{aligned}
& u_{r}(n, n-k)=\sum_{i=0}^{k} \sum_{j=-i}^{i}(-1)^{k+j}\left(\begin{array}{l}
n-i \\
k-i
\end{array}\right) s(n, n-i+j) s(n, n-i-j) r^{k-i}, \\
& U_{r}(n+k, n)=\sum_{i=0}^{k} \sum_{j=-i}^{i}(-1)^{i-j}\left(\begin{array}{c}
n+k \\
k-i
\end{array}\right) S(n+i+j, n) S(n+i-j, n) r^{k-i} .
\end{aligned}
$$

Proof From (44), (33), and (39), we get

$$
\begin{aligned}
u_{r}(n, n-k) & =(-1)^{k} \sigma_{k}\left(r, 1^{2}+r, 2^{2}+r, \ldots,(n-1)^{2}+r\right) \\
& =(-1)^{k} \sum_{i=0}^{k}\left(\begin{array}{l}
n-i \\
k-i
\end{array}\right) \sigma_{i}\left(0,1^{2}, \ldots,(n-1)^{2}\right) r^{k-i} \\
& =\sum_{i=0}^{k} \sum_{j=-i}^{i}(-1)^{k+j}\left(\begin{array}{l}
n-i \\
k-i
\end{array}\right) \sigma_{i+j}(0,1, \ldots, n-1) \sigma_{i-j}(0,1, \ldots, n-1) r^{k-i} \\
& =\sum_{i=0}^{k} \sum_{j=-i}^{i}(-1)^{k+j}\left(\begin{array}{l}
n-i \\
k-i
\end{array}\right) s(n, n-i+j) s(n, n-i-j) r^{k-i}
\end{aligned}
$$

From (45), (34), and (39),

$$
\begin{aligned}
U_{r}(n+k, n) & =h_{k}\left(r, 1^{2}+r, 2^{2}+r, \ldots, n^{2}+r\right) \\
& =\sum_{i=0}^{k}\left(\begin{array}{c}
n+k \\
k-i
\end{array}\right) h_{i}\left(0,1^{2}, \ldots, n^{2}\right) r^{k-i} \\
& =\sum_{i=0}^{k} \sum_{j=-i}^{i}(-1)^{i-j}\left(\begin{array}{c}
n+k \\
k-i
\end{array}\right) h_{i+j}(0,1, \ldots, n) h_{i-j}(0,1, \ldots, n) r^{k-i} \\
& =\sum_{i=0}^{k} \sum_{j=-i}^{i}(-1)^{i-j}\left(\begin{array}{c}
n+k \\
k-i
\end{array}\right) S(n+i+j, n) S(n+i-j, n) r^{k-i} .
\end{aligned}
$$

For example,

$$
\begin{aligned}
u_{r}(6,5) & =\sum_{i=0}^{1} \sum_{j=-i}^{i}(-1)^{1+j}\left(\begin{array}{c}
6-i \\
1-i
\end{array}\right) s(6,6-i+j) s(6,6-i-j) r^{1-i}=-6 r-55, \\
U_{r}(5,3) & =\sum_{i=0}^{2} \sum_{j=-i}^{i}(-1)^{i-j}\left(\begin{array}{c}
5 \\
2-i
\end{array}\right) S(3+i+j, 3) S(3+i-j, 3) r^{2-i} \\
& =10 r^{2}+70 r+147 .
\end{aligned}
$$

Remark 2 The term at $i=k$ in (47) gives the following identity, which was shown previously in [22]:

$$
u(n, n-k)=\sum_{j=-k}^{k}(-1)^{k+j} s(n, n-k+j) s(n, n-k-j)
$$


And the term at $i=k$ in (48) shows a new connection between $U(n, k)$ and $S(n, k)$ :

$$
U(n+k, n)=\sum_{j=-k}^{k}(-1)^{k-j} S(n+k+j, n) S(n+k-j, n) .
$$

\section{The $r$-central factorial matrices}

In the following, we consider the $r$-central factorial matrices with even indices of both kinds, then we obtain factorization of these matrices.

Definition 2 The $r$-central factorial matrices with even indices of the first kind $\mathcal{U}_{1}(n)$ and of the second kind $\mathcal{U}_{2}(n)$ are the $n \times n$ matrices defined by

$$
\mathcal{U}_{1}(n):=\mathcal{U}_{1}^{(r)}(n)=\left[u_{r}(i, j)\right]_{0 \leq i, j \leq n-1}
$$

and

$$
\mathcal{U}_{2}(n):=\mathcal{U}_{2}^{(r)}(n)=\left[\mathcal{U}_{r}(i, j)\right]_{0 \leq i, j \leq n-1} .
$$

For example, $\mathcal{U}_{1}(5)$ is given by

$$
\left[\begin{array}{ccccr}
1 & 0 & 0 & 0 & 0 \\
-r & 1 & 0 & 0 & 0 \\
r^{2}+r & -2 r-1 & 1 & 0 & 0 \\
-r^{3}-5 r^{2}-4 r & 3 r^{2}+10 r+4 & -3 r-5 & 1 & 0 \\
r^{4}+14 r^{3}+49 r^{2}+36 r & -4 r^{3}-42 r^{2}-98 r-36 & 6 r^{2}+42 r+49 & -4 r-14 & 1
\end{array}\right],
$$

and $\mathcal{U}_{2}(5)$ is given by

$$
\left[\begin{array}{ccccc}
1 & 0 & 0 & 0 & 0 \\
r & 1 & 0 & 0 & 0 \\
r^{2} & 2 r+1 & 1 & 0 & 0 \\
r^{3} & 3 r^{2}+3 r+1 & 3 r+5 & 1 & 0 \\
r^{4} & 4 r^{3}+6 r^{2}+4 r+1 & 6 r^{2}+20 r+21 & 4 r+14 & 1
\end{array}\right] .
$$

In particular, if $r=0$, we obtain the central factorial matrices with even indices of both kinds:

$$
\mathcal{A}_{1}(n)=[u(i, j)]_{0 \leq i, j \leq n-1} \text { and } \mathcal{A}_{2}(n)=[U(i, j)]_{0 \leq i, j \leq n-1}
$$

Note that the orthogonality property (13) is equivalent to the matrix equation

$$
\mathcal{U}_{1}(n) \mathcal{U}_{2}(n)=\mathcal{U}_{2}(n) \mathcal{U}_{1}(n)=\mathbf{I}
$$

with $\mathbf{I}$ being the $n \times n$ unit matrix. Hence, we have the identity

$$
\left(\mathcal{U}_{1}(n)\right)^{-1}=\mathcal{U}_{2}(n), \quad n \geq 1
$$


In fact, inverse relations are known for practically all special numbers, such as Whitney numbers and Stirling numbers (see $[6,7,25,26]$ ).

Recall that the generalized $n \times n$ Pascal matrix $\mathbf{P}_{n}[z]$ is defined as follows (see [5]):

$$
\mathbf{P}_{n}[z]=\left[\left(\begin{array}{c}
i \\
j
\end{array}\right) z^{i-j}\right]_{0 \leq i, j \leq n-1},
$$

with $\mathbf{P}_{n}=\mathbf{P}_{n}[1]$, the Pascal matrix of order $n$. For example,

$$
\mathbf{P}_{5}[z]=\left[\begin{array}{ccccc}
1 & 0 & 0 & 0 & 0 \\
z & 1 & 0 & 0 & 0 \\
z^{2} & 2 z & 1 & 0 & 0 \\
z^{3} & 3 z^{2} & 3 z & 1 & 0 \\
z^{4} & 4 z^{3} & 6 z^{2} & 4 z & 1
\end{array}\right]
$$

Moreover,

$$
\mathbf{P}_{n}^{-1}[z]=\mathbf{P}_{n}[-z]=\left[(-1)^{i-j}\left(\begin{array}{l}
i \\
j
\end{array}\right) z^{i-j}\right]_{0 \leq i, j \leq n-1} .
$$

From (40) and (42), we have the following factorization:

$$
\mathcal{U}_{1}(n)=\mathcal{A}_{1}(n) \mathbf{P}_{n}[-r], \quad n \geq 1,
$$

and

$$
\mathcal{U}_{2}(n)=\mathbf{P}_{n}[r] \mathcal{A}_{2}(n), \quad n \geq 1
$$

For example,

$$
\begin{aligned}
\mathcal{U}_{1}(5) & =\left[\begin{array}{ccccc}
1 & 0 & 0 & 0 & 0 \\
0 & 1 & 0 & 0 & 0 \\
0 & -1 & 1 & 0 & 0 \\
0 & 4 & -5 & 1 & 0 \\
0 & -36 & 49 & -14 & 1
\end{array}\right] \times\left[\begin{array}{ccccc}
1 & 0 & 0 & 0 & 0 \\
-r & 1 & 0 & 0 & 0 \\
r^{2} & -2 r & 1 & 0 & 0 \\
-r^{3} & 3 r^{2} & -3 r & 1 & 0 \\
r^{4} & -4 r^{3} & 6 r^{2} & -4 r & 1
\end{array}\right] \\
& =\mathcal{A}_{1}(5) \mathbf{P}_{5}[-r]
\end{aligned}
$$

and

$$
\begin{aligned}
\mathcal{U}_{2}(5) & =\left[\begin{array}{ccccc}
1 & 0 & 0 & 0 & 0 \\
r & 1 & 0 & 0 & 0 \\
r^{2} & 2 r & 1 & 0 & 0 \\
r^{3} & 3 r^{2} & 3 r & 1 & 0 \\
r^{4} & 4 r^{3} & 6 r^{2} & 4 r & 1
\end{array}\right] \times\left[\begin{array}{ccccc}
1 & 0 & 0 & 0 & 0 \\
0 & 1 & 0 & 0 & 0 \\
0 & 1 & 1 & 0 & 0 \\
0 & 1 & 5 & 1 & 0 \\
0 & 1 & 21 & 14 & 1
\end{array}\right] \\
& =\mathbf{P}_{5}[r] \mathcal{A}_{2}(5) .
\end{aligned}
$$




\section{Conclusions}

In this paper, we introduced the $r$-central factorial numbers with even indices of both kinds as extended versions of the central factorial numbers with even indices of both kinds. We derived the generating functions, some explicit expressions, and orthogonality relations for such special numbers. In addition, we showed the relations between such numbers and the central factorial numbers with even indices which were also interpreted in matrix forms involving Pascal matrices. Also, we showed the relations between such numbers and the Stirling numbers. Finally, as an application to probability, we gave the probability distribution of the unsigned $r$-central factorial numbers with even indices of the first kind.

\section{Acknowledgements}

The author is deeply grateful to the editor and referees for their helpful comments in improving the presentation and quality of the paper.

\section{Funding}

There is no funding for this article.

Availability of data and materials

Data sharing not applicable to this article as no data sets were generated or analysed during the current study.

Competing interests

The author declares that she has no competing interests.

Authors' contributions

The author wrote the first version of the manuscript and approved the final manuscript by herself.

\section{Publisher's Note}

Springer Nature remains neutral with regard to jurisdictional claims in published maps and institutional affiliations.

Received: 26 February 2020 Accepted: 10 June 2020 Published online: 17 June 2020

\section{References}

1. Acikgoz, M., Araci, S., Duran, U.: Some ( $p ; q)$-analogues of Apostol type numbers and polynomials. Acta Comment. Univ. Tartu Math. 23(1), 37-50 (2019)

2. Araci, S., Duran, U., Acikgoz, M.: On weighted q-Daehee polynomials with their applications. Indag. Math. 30, 365-374 (2019)

3. Bóna, M.: Combinatorics of Permutations. Chapman \& Hall/CRC, London (2004)

4. Butzer, P.L., Schmidt, K., Stark, E.L., Vogt, L.: Central factorial numbers; their main properties and some applications. Numer. Funct. Anal. Optim. 10(5\&6), 419-488 (1989)

5. Call, G.S., Velleman, D.J.: Pascal's matrices. Am. Math. Mon. 100, 372-376 (1993)

6. El-Desouky, B.S., Shiha, F.A.: A q-analogue of $\bar{\alpha}$-Whitney numbers. Appl. Anal. Discrete Math. 12, 178-191 (2018)

7. El-Desouky, B.S., Shiha, F.A., Shokr, E.M.: The multiparameter $r$-Whitney numbers. Filomat 33(3), 931-943 (2019)

8. Gelineau, Y., Zeng, J.: Combinatorial interpretations of the Jacobi-Stirling numbers. Electron. J. Comb. 17, R70 (2010)

9. Hardy, G.H., Littlewood, J.E., Pólya, G.: Inequalities. Cambridge University Press, Cambridge (1959)

10. Jang, L.-C., Kim, T., Kim, D.S., Kim, H.Y.: Extended r-central Bell polynomials with umbral calculus viewpoint. Adv. Differ Equ. 2019, 202 (2019)

11. Khan, N., Usman, T., Choi, J.: A new class of generalized polynomials associated with Laguerre and Bernoulli polynomials. Turk. J. Math. 43, 486-497 (2019)

12. Kim, D.S., Dolgy, D.V., Kim, D., Kim, T.: Some identities on $r$-central factorial numbers and $r$-central Bell polynomials. Adv. Differ. Equ. 2019, 245 (2019)

13. Kim, D.S., Dolgy, D.V., Kim, T., Kim, D.: Extended degenerate $r$-central factorial numbers of the second kind and extended degenerate r-central Bell polynomials. Symmetry 11(4), Article ID 595 (2019)

14. Kim, D.S., Kim, H.Y., Kim, D., Kim, T.: On r-central incomplete and complete Bell polynomials. Symmetry 11(5), Article ID 724 (2019)

15. Kim, T., Jang, L.-C., Kim, D.S., Kim, H.Y.: Some identities on type 2 degenerate Bernoulli polynomials of the second kind. Symmetry 12(4), Article ID 510 (2020)

16. Kim, T., Kim, D.S.: A note on central Bell numbers and polynomials. Russ. J. Math. Phys. 27(1), $76-81$ (2020)

17. Kim, T., Kim, D.S.: Degenerate polyexponential functions and degenerate Bell polynomials. J. Math. Anal. Appl. 487(2), $124017(2020)$

18. Kim, T., Kim, D.S.: Some identities of extended degenerate $r$-central Bell polynomials arising from umbral calculus. Rev. R. Acad. Cienc. Exactas Fís. Nat., Ser. A Mat. 114(1), Paper No. 1, 19 pp. (2020)

19. Kim, T., Kim, D.S., Jang, G.-W.: On central complete and incomplete Bell polynomials I. Symmetry 11(2), Article ID 288 (2019) 
20. Kim, T., Kim, D.S., Jang, G.-W., Kwon, J.: Extended central factorial polynomials of the second kind. Adv. Differ. Equ. 2019, 24 (2019)

21. Kwon, J., Kim, T., Kim, D.S., Kim, H.Y.: Some identities for degenerate complete and incomplete $r$-Bell polynomials. J. Inequal. Appl. 2020, 23 (2020)

22. Merca, M.: A special case of the generalized Girard-Waring formula. J. Integer Seq. 15, Article 12.5 .7 (2012)

23. Merca, M.: A note on the $r$-Whitney numbers of Dowling lattices. C. R. Acad. Sci. Paris, Ser. I 351, 649-655 (2013)

24. Merca, M.: Connections between central factorial numbers and Bernoulli polynomials. Period. Math. Hung. 73(2), 259-264 (2016)

25. Mezö, I., Ramírez, J.L.: The linear algebra of the $r$-Whitney matrices. Integral Transforms Spec. Funct. 26(3), 213-225 (2015)

26. Riordan, J.: Combinatorial Identities. Wiley, New York (1968)

27. Samuels, S.M.: On the number of successes in independent trials. Ann. Math. Stat. 36(4), 1272-1278 (1965)

28. Shattuck, M.: Generalizations of Bell number formulas of Spivey and Mezö. Filomat 30(10), 2683-2694 (2016)

29. Stirling, J.: Methodus differentialis: Sive Tractatus de Summatione et Interpolatione Serierum Inifinitarum. London (1730)

30. Wilf, H.S.: Generating Functionology. Academic Press/Harcourt Brace Jovanovich (1994)

\section{Submit your manuscript to a SpringerOpen ${ }^{\circ}$ journal and benefit from:}

- Convenient online submission

- Rigorous peer review

- Open access: articles freely available online

- High visibility within the field

- Retaining the copyright to your article

Submit your next manuscript at $\gg$ springeropen.com 